\title{
Gonadectomia em gatas impúberes. Técnica anestésica
}

Sandra MASTROCINQUE

Vivianne Higuchi

IMAGAWA

Tatiana Ferrante de

ALMEIDA ${ }^{1}$

Angelica Cecília

TATARUNAS

Julia Maria MATERA

Denise Tabacchi FANTONI

Correspondência para SANDRAMASTROCINQUE Rua Sampaio Viana, 688. Apto 131. 04004-002 - Paraíso - São Paulo - SP vivifonfon@uol.com.br

Recebido para publicação: 11/01/2006 Aprovado para publicação: 13/02/2006

1 - Faculdade de Medicina Veterinária e Zootecnia da Universidade de São Paulo, São Paulo - SP

\section{Resumo}

O objetivo deste estudo foi descrever e avaliar um protocolo anestésico em filhotes de gatas submetidas a gonadectomia. Foram estudadas 40 fêmeas, com 2 a 4 meses de idade, sem raça definida, encaminhadas para ovariohisterectomia. O protocolo anestésico empregado consistiu de pré-medicação com acepromazina e meperidina e a indução e manutenção anestésicas realizadas com tiletamina-zolazepam. Foram avaliadas freqüências cardíaca e respiratória, saturação periférica da oxihemoglobina, temperatura corpórea, qualidade do relaxamento de coto uterino e pedículos ovarianos, tempo cirúrgico e de recuperação anestésica, bem como qualidade da recuperação anestésica e outros efeitos adversos. Houve redução da freqüência respiratória e manutenção da cardíaca no período trans-operatório. Apenas dois animais necessitaram de suplementação com oxigênio e a qualidade da recuperação foi satisfatoriamente tranqüila. $\mathrm{O}$ relaxamento dos pedículos ovarianos e coto uterino foi adequado e não foram observados demais efeitos adversos. Diante dos resultados obtidos pode-se concluir que a técnica anestésica empregada foi segura e efetiva nos animais estudados.

\section{Introdução}

A superpopulação de cães e gatos é um problema social de cunho mundial. Frente ao grande número de animais que são submetidos à eutanásia diariamente com o propósito de controle populacional, procurou-se uma forma alternativa a esta medida e concluiu-se que a castração é um método importante de controle e a sua eficácia é maior quando os animais são castrados antes de atingirem a puberdade, desta forma impedindo a sua reprodução ${ }^{1}$.

As vantagens da gonadectomia incluem a presença de gordura abdominal mínima, permitindo excelente visualização das estruturas vasculares e reprodutivas; os vasos de tamanho diminuto tornam a hemostasia precisa, reduzindo o tempo operatório; a recuperação é extremamente rápida e a morbidade é baixa ${ }^{2}$.

Durante a cirurgia de pacientes pediátricos, deve-se estar atento para a hipotermia e hipoglicemia, as quais os filhotes são predispostos ${ }^{1,2}$, bem como ao período de jejum ao qual o animal é submetido e ao excesso de soluções antisépticas nos pêlos ${ }^{2}$. Geralmente os pacientes pediátricos se recuperam rapidamente da anestesia e devem ser alimentados em 1 a 2 horas após o procedimento cirúrgico ${ }^{3}$.

Por outro lado, os tecidos pediátricos são friáveis e, portanto devem ser manipulados delicadamente, considerando que o pequeno volume sanguíneo torna a hemostasia meticulosa de extrema importância ${ }^{1,4}$.

A técnica, quando realizada adequadamente é segura, produzindo estresse mínimo aos pacientes pediátricos ${ }^{1}$.

Perante o tamanho diminuto dos animais e conseqüentemente de suas estruturas intra-abdominais e da necessidade de técnica cirúrgica-anestésica que otimize o procedimento, avaliou-se a exeqüibilidade do uso de gancho de castração ${ }^{5}$ e a qualidade da técnica anestésica empregada, através da mensuração de parâmetros fisiológicos, 
correlacionando estes dados ao grau de relaxamento dos pedículos ovarianos e do coto uterino, bem como a recuperação dos pacientes em termos de qualidade e tempo em gatas filhotes de 2 a 4 meses de idade.

Dentre os cuidados a serem tomados com os filhotes, é importante evitar o estresse destes pacientes, minimizando sua manipulação, e se possível deixando-os em ambiente calmo e com temperatura adequada. A dor e demais fatores de estresse ocasionados pelo ato cirúrgico e anestésico devem ser levados em conta, pois mesmo pacientes muito jovens são capazes de produzir respostas deletérias aos fatores causadores de estresse, uma vez que as vias nociceptivas e sistema funcional para resposta ao estresse estão presentes desde o nascimento ${ }^{6}$. Além disto, o jejum não deve ultrapassar 8 horas em pacientes pediátricos e 3 horas em animais com menos de 10 semanas de idade, uma vez que estes pacientes estão mais predispostos a apresentarem hipoglicemia ${ }^{7,8}$.

Posto isto, o objetivo do trabalho foi o de avaliar e descrever um protocolo anestésico em filhotes de gatas submetidas à ovariohisterectomia.

\section{Materiais e Métodos}

Foram utilizadas 40 gatas, com 2 a 4 meses de idade, sem raça definida, pesando entre 1,0 e 2,5 $\mathrm{kg}$ atendidas no Hospital Veterinário da Faculdade de Medicina Veterinária e Zootecnia da Universidade de São Paulo. Após anamnese, inspeção e exame físico atestando bom estado geral, prévia

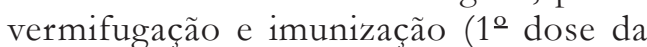
vacina tríplice felina), os animais foram submetidos a jejum alimentar de 4 horas e hídrico de 1 hora previamente ao procedimento cirúrgico.

Todos os animais foram submetidos à ovariohisterectomia, realizada sempre pelo mesmo cirurgião por meio de técnica descrita com utilização do gancho de castração ${ }^{5}$.

O protocolo anestésico ao qual os animais foram submetidos foi baseado no uso de acepromazina (Acepran ${ }^{\circledR}$, Univet) na dose de $0,2 \mathrm{mg} / \mathrm{kg}$ e meperidina na dose de $8 \mathrm{mg} / \mathrm{kg}$, associados na mesma seringa e aplicados pela via intramuscular como medicação pré-anestésica (MPA). Os animais foram mantidos em ambiente calmo e tranqüilo e com pouca iluminação, evitandose a manipulação excessiva e, decorridos 10 minutos da aplicação da MPA, o acesso venoso periférico foi obtido por meio da venopunção da veia cefálica com a utilização do cateter $24 \mathrm{G}$ e iniciada fluidoterapia com Ringer lactato na taxa de $5 \mathrm{ml} / \mathrm{kg} /$ hora para a manutenção da volemia e reposição de eletrólitos.

A indução e manutenção anestésica foram realizadas com a utilização da associação de tiletamina e zolazepam $\left(\right.$ Zoletil $\left.^{\circledR}\right)$, na dose de $6 \mathrm{mg} / \mathrm{kg}$ pela via intravenosa (lentamente e diluído em $3 \mathrm{ml}$ de solução salina) e reaplicada durante o ato cirúrgico na dose de $3 \mathrm{mg} / \mathrm{kg}$, caso o animal apresentasse sinais de superficialização da anestesia como aumento das freqüências cardíaca e/ou respiratória na taxa de $20 \%$ do valor basal.

Os parâmetros fisiológicos avaliados foram: freqüências cardíaca e respiratória e saturação periférica de oxihemoglobina, no período pré-operatório (anteriormente a aplicação da MPA), logo após a indução anestésica e a cada 5 minutos após a indução anestésica (Figura 1). Durante o período trans-operatório empregou-se o monitor multiparamétrico $\left(\right.$ Dixtal $\left.^{\mathbb{Q}}\right)$ para avaliação da freqüência e ritmo cardíacos, bem como para obtenção dos valores de saturação periférica da oxihemoglobina (Figura 1). Caso os animais apresentassem diminuição nos valores da saturação periférica da oxihemoglobina, eram submetidos à inalação de oxigênio via máscara facial.

Mensurou-se a temperatura retal no período pré-operatório (anteriormente à aplicação da MPA) e ao final do procedimento cirúrgico.

Avaliou-se também, de forma subjetiva e sempre pelo mesmo cirurgião o 


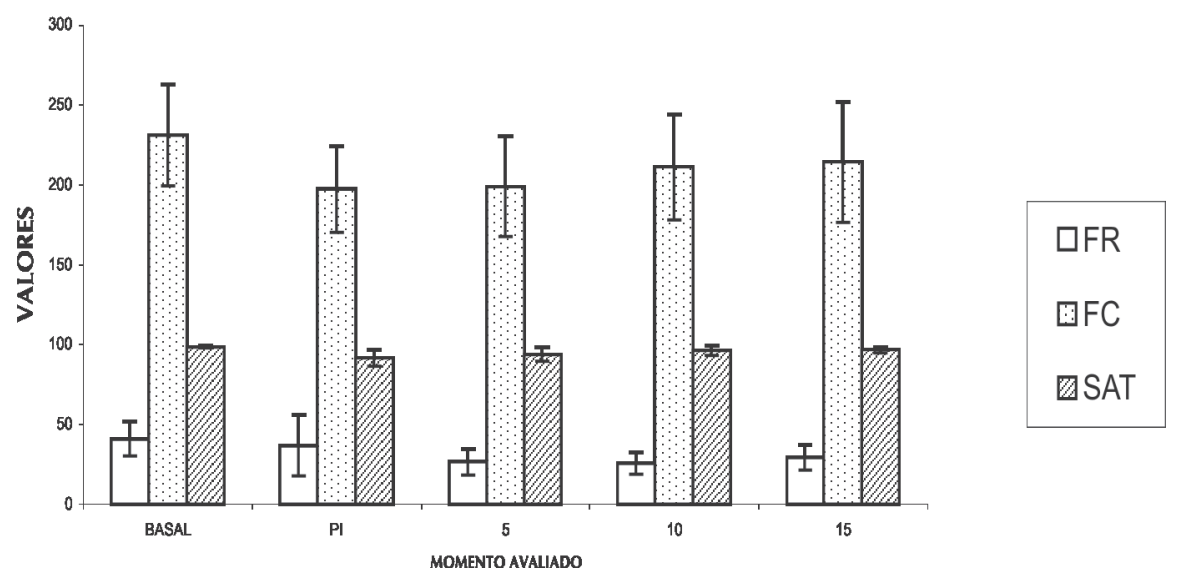

Figura 1 - Comportamento das frequiências cardíaca e respiratória e saturação periférica da oxihemoglobina ao longo do período de avaliação nos animais estudados $(\mathrm{N}=40)$

relaxamento dos pedículos ovarianos e do coto uterino, o qual foi graduado em ótimo, bom e regular.

Foram registrados os períodos cirúrgico e de recuperação anestésica. O período cirúrgico foi considerado desde a incisão até o final da sutura de pele, e o período de recuperação anestésica entre o término da sutura de pele e o início de apresentação de meneios de cabeça, reflexo interdigital ou decúbito esternal. Durante a recuperação da anestesia deu-se especial atenção à presença de excitação, caracterizada por movimentos de pedalar, vocalização ou rigidez muscular.

As complicações anestésicas pré, trans e pós-operatórias foram consideradas, como sendo: alterações de freqüência cardíaca e/ou respiratória em torno de $20 \%$ do valor basal, arritmias cardíacas, alteração da saturação periférica da oxihemoglobina inferior a $90 \%$.

A analgesia pós-operatória foi realizada com a administração de tramadol $\left(\right.$ Tramal - Searle $\left.{ }^{\circledR}\right)$ pela via subcutânea na dose de $1 \mathrm{mg} / \mathrm{kg}$, logo após o término do procedimento cirúrgico.

Durante o período pós-operatório, os animais foram mantidos em ambiente tranqüilo e aquecido e alimentados após 1 hora, se acordados.

Os resultados (freqüência cardíaca, freqüência respiratória, saturação de oxihemoglobina, temperatura corporal, tempo cirúrgico e tempo de recuperação da anestesia) foram submetidos à análise de variância (ANOVA), para avaliar os efeitos do tempo sobre as mesmas. Onde o efeito foi significativo, foi realizado teste de Tukey para comparação de médias, sendo adotado nível de significância de 5\% para todos os testes.

\section{Resultados}

As avaliações das freqüências cardíaca e respiratória e da saturação periférica da oxihemoglobina nos momentos préoperatório, imediatamente após a indução, bem como 5, 10, 15 e 20 minutos de transoperatório encontram-se dispostos na tabela 1.

Os valores da média e do desvio padrão da temperatura corpórea inicial e final foram $38,15^{\circ} \mathrm{C} . \pm 0,63^{\circ} \mathrm{C}$ e $36,67^{\circ} \mathrm{C} \pm$ $0,54^{\circ} \mathrm{C}$, respectivamente.

A técnica anestésica empregada possibilitou a realização do procedimento 
cirúrgico em todos os animais estudados. Durante a cirurgia observou-se ótimo relaxamento dos pedículos e coto uterino em $85 \%(n=34)$ dos animais, bom em 12,5\% $(n=5)$ e regular em $2,5 \%(n=1)$.

O período cirúrgico médio e desvio

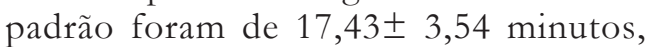
sendo o menor período cirúrgico de 13 minutos e o maior de 30 minutos.

Em 10 dos 40 animais estudados (25\%) houve necessidade de dose adicional do fármaco indutor (tiletamina-zolazepam) para manutenção anestésica. Nos demais, a dose inicial para indução da anestesia foi suficiente para realização do procedimento cirúrgico.

O período médio para que os animais apresentassem sinais de recuperação (em decúbito esternal, movendo a cabeça e com reflexo interdigital presente) foi de 15,6 \pm 2,12 minutos. Durante a recuperação da anestesia dois (5\%) animais apresentaram movimentos de pedalar e rigidez muscular. A vocalização foi ausente em todos (100\%) dos animais.

Não foram observados outros efeitos adversos durante os períodos pré, trans e pós-operatórios.

\section{Discussão}

A grande necessidade do controle populacional de cães e gatos deve estar associada à busca de técnicas anestésicas seguras e efetivas, uma vez que a esterilização dos animais anteriormente à puberdade é mais efetiva no que tange ao controle reprodutivo dos animais, porém exige maior cuidado por parte da equipe cirúrgica e anestésica ${ }^{1}$.

No estudo em discussão pôde-se observar que a técnica anestésica proposta possibilitou a realização da gonadectomia nas fêmeas, com bom relaxamento dos pedículos ovarianos e coto uterino, diferindo do observado por Faggella e Aronsohn ${ }^{1}$, os quais relataram que a associação de tiletamina-zolazepam foi efetiva no que diz respeito a analgesia e relaxamento para esterilização de machos, mas não das fêmeas, as quais necessitaram de complementação com anestésico inalatório. Deve-se ressaltar que a associação de opióides aos anestésicos injetáveis melhora a qualidade da técnica, além de reduzir as doses destes últimos, diminuindo os efeitos indesejáveis destes agentes. A necessidade de menores doses foi verificada neste estudo, onde a associação da tiletamina-zolazepam à meperidina possibilitou um procedimento cirúrgicoanestésico com baixas doses do anestésico para manutenção da anestesia. De fato, em $75 \%$ dos animais a cirurgia foi realizada com apenas uma dose do agente indutor. A associação demonstrou ainda ser segura para o sistema cardiovascular, pois não foram observados valores baixos da freqüência cardíaca ou alterações de ritmo cardíaco em nenhum dos animais estudados. O opióide empregado, a meperidina, causa depressão

Tabela 1 - Média e desvio padrão dos valores de freqüência cardíaca (batimentos por minuto), freqüência respiratória (movimentos por minuto) e saturação periférica da oxihemoglobina (\%) nos tempos pré-operatório (basal), imediatamente após a indução anestésica (indução) e nos tempos 5, 10 e 15 minutos trans-operatórios. São Paulo, 2004

\begin{tabular}{cccccc}
\hline & Valor basal & Indução & 5 minutos & 10 minutos & 15 minutos \\
\hline FC(bpm) & $231 \pm 31,82$ & $197,5 \pm$ & $199,12 \pm$ & $211,45 \pm$ & $214,48 \pm$ \\
& & 27,35 & 31,53 & 32,90 & 37,53 \\
FR(mpm) & $40,7 \pm 10,61$ & $33,81 \pm$ & $26,43 \pm 8,12$ & $25,53 \pm 6,61$ & $29,17 \pm 7,87$ \\
SAT(\%) & $98,4 \pm 0,71.0$ & $91,54 \pm 5,35$ & $93,78 \pm 4,31$ & $96,0 \pm 3,15$ & $96,5 \pm 1,68$ \\
\hline
\end{tabular}

FC: Freqüência cardíaca

FR: Freqüência respiratória

bpm: batimentos por minuto

mpm: movimentos por minuto

SAT: saturação periférica de oxihemoglobina 
miocárdica ${ }^{9,10}$ mínima, assim como a associação tiletamina-zolazepam, a qual pode ainda levar a incrementos na freqüência cardíaca, devido a seu efeito simpatomimético ${ }^{11}$. Estas elevações podem ser amenizadas após prétratamento com acepromazina $1^{12}$, fato que pode ter ocorrido no presente estudo. Apesar de alguns autores recomendarem a associação de antico-linérgicos, como a atropina, na medicação pré-anestésica de animais jovens para evitar bradicardia ${ }^{13}$, este fármaco não foi empregado no estudo em tela, uma vez que não foi observada a redução da freqüência cardíaca em nenhum dos animais avaliados. A diminuição significativa da freqüência respiratória após o início da anestesia, em relação ao valor basal, já era prevista e relacionada à depressão dos centros respiratórios pela tiletamina e pelo zolazepam $^{14,15}$. O grau de depressão respiratória após o emprego de tiletaminazolazepam em gatos parece ser dosedependente, e caracterizado por padrão respiratório apnêustico que se normaliza 10 a 15 minutos após a administração do fármaco ${ }^{16}$, sendo este um achado deste estudo, pois a freqüência respiratória e a saturação periférica da oxihemoglobina se normalizaram 15 minutos após a indução anestésica.

Os animais apresentaram recuperação bastante tranqüila, provavelmente devido à associação de um agente tranqüilizante (acepromazina) com um analgésico opióide (meperidina) no período pré-anestésico, concordando com as citações da literatura ${ }^{12,15}$, e possibilitando uma analgesia ${ }^{6}$ satisfatória no pós-operatório imediato.

A hipotermia apresentada pelos animais se deve ao fato de que pacientes jovens possuem elevada superfície corpórea, sistema termoregulador imaturo, pouca capacidade de se aquecer através de tremores e reduzida gordura subcutânea ${ }^{17,8}$, além destes fatores serem agravados durante a anestesia, devido à depressão da termorregulação e vasodilatação causada pelos agentes anestésicos. A hipotermia pode acarretar bradicardia, elevando o período de eliminação dos fármacos anestésicos, além de retardar a recuperação dos pacientes ${ }^{9,13}$, porém, tais efeitos não foram observados neste estudo, talvez pelo curto período de hipotermia que os pacientes sofreram.

\section{Conclusão}

Com base nos resultados obtidos, pode-se concluir que a técnica anestésica empregada foi segura e efetiva, permitindo analgesia e relaxamento cirúrgico adequados para a realização da ovariohisterectomia em felinas filhotes.

\section{Kittens gonadectomy. Anesthetic technique}

\section{Abstract}

The aim of this study was to evaluate an anesthetic protocol in female kittens to perform gonadectomy. Forty female kittens with ages varying from 2 to 4 months old, mixed breed were used. They were premedicated with acepromazina plus meperidina and the induction and maintenance of anesthesia were performed with tiletaminezolazepam. Variables measured were: cardiac and respiratory rates, oxyhemoglobin saturation, temperature, surgical relaxation, surgical and recovery times, recovery and undesirable effects. There was a reduction of respiratory rate after induction of anesthesia and cardiac rate remained stable during anesthesia period. Two animals needed oxygen therapy by facial mask and animals' recovery was smooth. The surgical relax was obtained in all animals and there were no other side effects. Based on these results it can be concluded that the anesthetic procedure was safe and effective to perform gonadectomy in female kittens.
Key-words:

Gonadectomy.

Cat.

Kittens.

Anesthesia.

Ovariohysterectomy. 


\section{Referências}

1 FAGGELLA, A. M.; ARONSOHN, M. G. Anesthetic techniques for neutering 6 to 14 week year's old kittens. Journal of American Veterinary Medical Association, v. 202, p. 56-62, 1993

2 STUBBS, W. P.; BLOOMBERG, M. S.; SCRUGGS, S. L.; SHILLE, V. M.; LANE, T. J. Effects of prepuberal gonadectomy on physical and behavioral development in cats. Journal of American Veterinary Medical Association, v. 209, n. 11, p. 1864-1871, 1996.

3 HOWE, L. M.; SLATER, M. R.; BOOTHE, H. W.; HOBSON, H. P.; FOSSUM, T. W.; SPAN, A. C.; WILKIE, W. S. Long-term outcome of gonadectomy performed at an early age or traditional age in cats. Journal of American Veterinary Medical Association v. 217, n. 11, p. 1661-5, 2000.

4 STUBBS, W. P.; BLOOMBERG, M. S. Implications of earlu neurtering in the dog and cat. Seminars in Veterinary Medical Surgery: Small Animals, v. 10, n. 1, p. 8-12, 1995.

5 TATARUNAS, A. C.; MATERA, J. M.; MASTROCINQUE, S.; IMAGAWA, V. H.; ALMEIDA, T. F.; FANTONI, D. T. Gonadectomia em gatas: técnica cirúrgica. Brazilian Journal Veterinary Research and Animal Science, v. 41, p. 184-185, 2004. Suplemento.

6 HICKEY P. R. Stress responses in pediatric patients. Seminars in Anesthesia, v. 12, n. 1, p. 1-7, 1993.

7 HOSGOOD, G. Surgical and anesthetic managent of puppies and kittens. The Compendium of Continuing Education: Small Animal Practice, v. 14, n. 3, p. 345 357, 1992.

8 KUSTRITZ, M. V. R Early spay-neuter in the dog and cat. Veterinary Clinics of North America: Small Animal Practice, v. 29, n. 4, p. 935-43, 1999

9 SCHIEBER, R. A; STILLER, R. L.; COOK D. R. Cardiovascular and pharmacodynamic effects of high dose of fentanyl in newborn piglets. Anesthesiology, v. 63, p. 166-171, 1985.

10 YASTER, M.; KOEHLER, R. C.; TRAYSTMAN, R. J. Effects of fentanyl on peripheral and cerebral hemodynamics in neonatal lambs. Anesthesiology, v. 66 , p. 524-530, 1987.

11 LIN, H. C.; THURMON, J. C.; BENSON, G. J.; TRANQUILLI, W. J. Telazol - a review of its pharmacology and use in veterinary medicine. Journal of Veterinary Therapeutics, v. 16, p. 383-418, 1992.

12 DONALDSON, L. L.; MCGRANTH, C. J.; TRACY, C. H. Testing low doses of intravenous Telazol in canine practice. Veterinary Medicine, v. 84, p. 1202-7, 1989.

13 PADDLEFORD, R. R. Anesthetic considerations in patients with preexistent problems or conditions. In: PADDLEFORD, $R, R$. Manual of small animal anesthesia. 2 ed. Philadelphia: W.B. Saunders, 1999. p. 267-317.

14 FANTONI, D. T * CORTOPASSI, S. R. G . BERNARDI, M. M. Anestésicos intravenosos e outros parenterais In: SPINOSA, H. S.; GÓRNIAK, S. L. BERNARD, M. M. Farmacologia aplicada à medicina veterinária. Rio de Janeiro: Guanabara Koogan, 1996. p. $105-114$

15 PABLO, L. S.; BAILEY, J. E. Etomidate and Telazol. Veterinary Clinics of North America: Small Animal Practice, v. 29, n. 3, p. 779-791, 1992.

16 TRACY, C. H.; SHORT, C. E.; CLARK, B. C. Comparing the effects of intravenous and intramuscular administration of Telazol. Veterinary Medicine, v. 83 p. 104-111, 1988.

17 GRANDY, J. L.; DUNLOP, C. I. Anesthesia of pups and kittens. Journal of American Veterinary Medical Association, v. 198, n. 7, p. 1244-1249, 1991.

18 PALMER, C.; VANUCCI, R. R.; CHRISTENSEN, M. A. Regional cerebral blood flow and glucose utilization during hypothermia in newborn dogs. Anesthesiology, v. 71, p. 730-737, 1989.

19 CORTOPASSI, S. R. G. anestesia pediátrica. In FANTONI, D. T.; COROTPASSI, S. R. G. Anestesia em Cães e Gatos. 1ed. São Paulo : Rocca, 1992. p.215-21. 